$16 \mid 2020$

Les marges créatrices : intellectuel.le.s afrodescendant.e.s et indigènes auX Amériques, XIX-XXe siècle

\title{
Catherine Rottenberg, The Rise of Neoliberal
}

\section{Feminism}

New York, Oxford University Press, 2018, 239 pages

Irène Favier

\section{OpenEdition}

\section{Journals}

Édition électronique

URL : http://journals.openedition.org/ideas/8502

DOI : 10.4000/ideas.8502

ISSN : 1950-5701

\section{Éditeur}

Institut des Amériques

\section{Référence électronique}

Irène Favier, "Catherine Rottenberg, The Rise of Neoliberal Feminism », IdeAs [En ligne], 16 | 2020, mis en ligne le 01 octobre 2020, consulté le 18 octobre 2020. URL : http://journals.openedition.org/ideas/ 8502 ; DOI : https://doi.org/10.4000/ideas.8502

Ce document a été généré automatiquement le 18 octobre 2020

\section{(c) (i) (3)}

IdeAs - Idées d'Amériques est mis à disposition selon les termes de la licence Creative Commons Attribution - Pas d'Utilisation Commerciale - Pas de Modification 4.0 International. 


\title{
Catherine Rottenberg, The Rise of Neoliberal Feminism
}

\author{
New York, Oxford University Press, 2018, 239 pages
}

Irène Favier

\section{RÉFÉRENCE}

Catherine Rottenberg, The Rise of Neoliberal Feminism, New York, Oxford University Press, 2018, 239 pages.

1 Julia, Ursula, Erika: trois personnages d'une telenovela péruvienne ${ }^{1}$ incarnent des attachements, des tiraillements et des dilemmes contemporains que la théorie féministe invite à analyser avec circonspection. Julia, enseignante en maternelle, arbore régulièrement des tee-shirts aux slogans favorables à la solidarité féminine et à l'«empouvoirement». Tandis qu'on apprend d'elle qu'elle est lectrice de Simone de Beauvoir, durant les coupures publicitaires l'actrice qui interprète Julia fait la promotion de marques de shampoing et de boisson gazeuse. Son amie Ursula, fille d'un délinquant recherché pour corruption, entreprend de conjurer le stigmate social légué par son père en tirant partie de sa formation en marketing dans une université étasunienne de la Ivy League: de haute lutte, elle obtient un poste dans une entreprise de Lima, où elle ne compte pas ses heures, passe avec difficulté sur les commentaires sexistes dont elle fait occasionnellement l'objet, et en oublie les événements censés ponctuer sa vie familiale, dans l'espoir d'une promotion interne à l'entreprise. Elle y fait recruter comme gérante Erika, sa voisine plus âgée d'une trentaine d'années, qui exprime ses inquiétudes quant à l'état de sa fertilité ; on la voit dans son bureau, sur le point de s'injecter le traitement hormonal préalable à la congélation des ovocytes.

2 À leur façon, ces trois personnages suggèrent l'importation dans la culture télévisuelle latino-américaine d'une phraséologie féministe, qu'on pourrait hâtivement attribuer à l'influence de la quatrième vague venue d'Amérique latine (or à aucun moment la série ne mentionne l'enjeu abortif) ou à une importation transatlantique quoique tardive 
d'une seconde vague beauvoirienne (or les trois vies sont largement guidées par l'objectif d'une mise en couple et nul obstacle structurel à leur cheminement en tant que personnes n'est évoqué), et que l'ouvrage de Catherine Rottenberg invite plutôt à appréhender sous l'angle du développement contemporain d'un féminisme néolibéral. Centré sur les Etats-Unis, cet ouvrage n'en aide pas moins à analyser la production culturelle dominante en vigueur dans des espaces où, comme au Pérou, l'hégémonie étatsunienne demeure incontestée, comme le révèle aisément un survol des pages culturelles des quotidiens nationaux.

Rottenberg mobilise elle aussi la production télévisuelle pour mettre en exergue un double phénomène qui revêt aux États-Unis une forme particulièrement prononcée et qui y trouve sa source: d'une part le regain de respectabilité de la cause féministe, promue jusque dans le show-business par des figures telles que Beyoncé et Emma Watson et consacré ce faisant au rang de féminisme mainstream, phénomène abondamment commenté par la théorie féministe et souvent légitimement analysé avec suspicion; d'autre part et de façon concomitante, la promotion à l'adresse de femmes « en responsabilité » d'un équilibre heureux entre vie professionnelle et vie familiale, selon une logique mise en image par la série largement diffusée The Good Wife. Plus de quête effrénée du conjoint, l'heure est à la recherche d'un graal renouvelé : celui d'un agenda permettant de s'épanouir au travail tout en profitant de ses enfants. Tandis que les jeunes femmes en cours de formation - c'est à dire ayant accès à la coûteuse éducation supérieure - ont été invitées dans les années 2000 à embrasser la hookup culture (ou culture du «coup d'un soir»), les révélations d'agressions sexuelles chroniques sur les campus ont mis en sourdine cet aspect de ce discours féministe d'un type nouveau. Désormais, la figure privilégiée de la production culturelle hégémonique a un poste "à responsabilités ", elle a eu des enfants, et doit trouver en elle-même les ressources - d'abord mentales - pour slalomer entre ses exigences également importantes et atteindre le but supposé de toute existence humaine : le bonheur.

Selon Rottenberg, il faut voir dans ce glissement de focale, de la jeune femme priorisant ses études vers la femme accomplie parce que mère et professionnelle, les deux faces d'un rapport au temps bien particulier : la première est invitée à retarder son entrée dans la procréation - la "futurité » néolibérale -, la seconde à s'immerger dans le temps présent de peur qu'il ne lui glisse entre les mains, les enfants grandissant sans qu'elle risque de s'en apercevoir - l'injonction de l'«ici et maintenant " héritière du New Age. Au-delà de la production télévisuelle et des mommy blogs, ce rapport au temps se déploie au travers d'une série de publications grand public, tantôt figurant en librairies au rayon de la littérature dite de self-help, tantôt - l'un n'excluant pas l'autre registre - se réclamant ouvertement du genre du manifeste féministe. Il s'agit de l'article publié par Anne-Marie Slaughter (Why Women Still Can't Have It All, 2012), des livres de Sheryl Sandberg (Lean In: Women, Work, and the Will to Lead, 2013), mais aussi d'Ivanka Trump (Women who work, 2017) et de Megyn Kelly (Settle for more, 2016). Si la presse a eu tendance à souligner leurs divergences, Rottenberg soutient que ce traitement médiatique tient davantage aux positionnements politiques préalables des auteures qu'aux profondes convergences internes qu'une analyse sérieuse des ouvrages conduit, au contraire, à mettre en lumière.

5 C'est avec sérieux que Rottenberg invite à lire ces textes dont elle estime que la critique aurait tort de leur refuser le qualificatif de féministe. Nulle ne peut en effet, précise-telle, sanctuariser la définition de ce terme sans courir le risque de la cantonner à une 
version issue de la seconde vague, donc datée, et de fermer ce faisant la porte à des contestations (et enrichissements) démocratiques ultérieures. Rottenberg soutient que le discours porté par ces textes relève bien d'un type de féminisme particulier, distinct d'une posture hypocrite se parant des oripeaux de la seconde vague pour, in fine, la " trahir » - distinct aussi, selon elle, d'un simple héritage de cette deuxième vague poussée jusque dans sa logique ultime. Elle prend pour exemple la question du travail domestique, rendu invisible par une partition de l'espace constitutive à l'avènement de la modernité opposant le public (tacitement masculin) au privé (tacitement féminin), et mis en lumière en 1989 par l'ouvrage de Arlie Russell Hochschild The Second Shift, réédité en 2012. Ainsi pour Rottenberg, si la seconde vague a dévoilé par ce type d'ouvrages, une dichotomie à l'œuvre dans la gestion sociale de la production et de la reproduction, le féminisme néolibéral s'emploie quant à lui à faire exploser cette distinction, en convertissant le sujet féminin en entrepreneure de son destin professionnel et personnel.

6 Sur ce point précis de la discontinuité entre seconde vague et féminisme néolibéral, l'analyse de Rottenberg diverge de celle de Nancy Fraser, qui voit dans l'avènement du féminisme néolibéral actuel une conséquence du détournement de la seconde vague de l'analyse matérialiste - toutes deux convergeant en revanche sur l'identification du féminisme néolibéral comme un phénomène radicalement nouveau. Or, si la démonstration de Rottenberg est convaincante à bien des égards et si sa réticence à attribuer à la seconde vague une culpabilité trop lourde dans l'avènement de la situation actuelle est louable, on peut continuer de s'interroger. Par exemple, et outre le penchant / pêché de la seconde vague privilégiant les luttes pour la reconnaissance aux luttes pour la redistribution mis en lumière par les travaux de Fraser, la place conférée à la citoyenneté par ces deux moments du féminisme interroge. En effet, dans le portrait-robot fait par le féminisme néolibéral de toute femme dotée de valeur et de ses aspirations restreintes aux seules sphères professionnelle et familiale et dépourvue de toute « aspiration » à la citoyenneté militante, n'y a-t-il pas là un héritage amer de la seconde vague, né de sa revendication pour ainsi dire professionnalo-centrée alors même que cette revendication était portée par une praxis militante? La question me semble se poser - celle, autrement dit, d'un paradoxe de la seconde vague, qui a promu l'extraction des femmes de la seule sphère domestique au profit d'un investissement dans le travail via la citoyenneté (c'est à dire par le biais de pratiques citoyennes revendicatives). Or si ces deux expressions d'une existence publique pour les femmes travail et activisme - partagent un même espace qualifié de public et ont en commun de se distinguer de la sphère reproductive / intime / privée, l'investissement citoyen semble avoir été progressivement invisibilisé au profit du gain d'autonomie financière rendu possible par la mise sur le marché du travail productif donc rémunéré. Cette question de l'occultation précoce de la citoyenneté féministe me semble se poser avant l'irruption de l'ère néolibérale.

7 Sur l'analyse du travail de la subjectivité par le néolibéralisme, Rottenberg emprunte à Wendy Brown certaines de ses démonstrations majeures, notamment celle qui appréhende le néolibéralisme, bien au-delà de ses seules injonctions économiques, comme un principe d'organisation du social colonisant des domaines croissants de l'existence et jusqu'à l'intimité du sujet. Ivanka Trump invite ainsi à investir tous les pans de sa vie à l'aune d'un calcul coût-avantage préalable - de la recherche du conjoint à la prévision des moments propices à la constitution d'un futur album de famille doté de valeur -, jusqu'aux domaines récréatif et amical. Une telle colonisation fait exploser 
les définitions spatiales en vigueur... pour mieux dépolitiser toute analyse de la condition féminine.

Ce discours n'est pas dépourvu de failles, suggère Rottenberg : s'il parvient à convertir les femmes en ressource humaine générique ("generic human capital») et les inégalités structurelles de genre en une question purement individuelle, la question de la reproduction de la main d'œuvre demeure un enjeu non résolu quoique retardé grâce aux moyens techniques. Il laisse également dans l'ombre la population - quasi exclusivement féminine, largement racialisée et déconsidérée en tant que peu bankable - en charge du travail de care relatif à l'entretien de la famille. Enfin et surtout (Rottenberg s'appuie ici sur les derniers travaux de Judith Butler) à la faveur des dérégulations sociales et financières et des désaffiliations que le néolibéralisme entraîne, une population croissante se trouve précipitée dans la précarité. Une telle condition, quoiqu'elle concerne des situations fort diverses, n'en constitue pas moins un point de rassemblement potentiel pour un féminisme radicalement alternatif, constitué non plus comme un mantra individuel, volontariste et excluant, réservé au « $1 \% »$, mais en un mouvement de masse ouvertement tourné vers la justice sociale.

Peut-être les suggestions conclusives sont-elles les plus hâtives de l'ouvrage ; elles ne font certes qu'esquisser des pistes générales pour une réappropriation du féminisme sous un angle unitaire, qui ne rechignerait pas au clivage politique, ni ne craindrait pas de s'emparer de l'appareil d'État pour le transformer en profondeur. Pour une interrogation sur la stratégie à adopter pour faire advenir ce féminisme pour les $99 \%$, on pourra lire Aurore Koechlin, Cinzia Arruzza, Tithi Bhattacharya et Nancy Fraser. Mais faire cette critique à l'ouvrage de Rottenberg serait injuste, tant il s'emploie à une tâche préalable impérative et jusqu'alors remarquablement laissée en attente : celle consistant à identifier le phénomène bien particulier du féminisme néolibéral, à le défaire de ses dehors fallacieusement unanimistes, class- et color-blind, et à en dévoiler les ressorts jusque dans ses heures de grande diffusion télévisuelle. Merci à l'ouvrage pour ça!

\section{BIBLIOGRAPHIE}

Slaughter, Anne-Marie, “Why Women Still Can't Have It All”, The Atlantic, 2012. https:// www.theatlantic.com/magazine/archive/2012/07/why-women-still-cant-have-it-all/309020/ page consultée le 10 septembre 2020.

Sandberg, Sheryl, Lean In: Women, Work, and the Will to Lead, New York, Knopf Doubleday Publishing Group, 2013.

Trump, Ivanka, Women who work, Rewriting the Rules for Success, New York, Penguin, 2017.

Kelly, Megyn, Settle for more, New York, HarperCollins Publishers, 2016.

Russell Hochschild, Arlie, The Second Shift, Working Families and the Revolution at Home, New York, Penguin, 2003. 
NOTES

1. Los Vilchez, produite par ProTV Producción pour América TV, diffusée en 2019 et 2020.

\section{AUTEURS}

\section{IRÈNE FAVIER}

Université de Grenoble / LARHRA 